\title{
Two malignant solitary fibrous tumors in one kidney: Case report and review of the literature
}

\author{
GUANGNING ZHAO, GANG LI and RUIFA HAN
}

Department of Urology, Second Hospital of Tianjin Medical University, Tianjin Institute of Urology, Tianjin 300211, P.R. China

Received May 8, 2012; Accepted August 3, 2012

DOI: $10.3892 / \mathrm{ol} .2012 .858$

\begin{abstract}
Malignant solitary fibrous tumors of the kidney are very rare. Two tumors in one kidney is particularly rare and has not been previously reported. Due to the non-specific clinical symptoms, it is difficult but also very significant to give a definite diagnosis. Here, we report a case of two renal masses in one kidney. A 56-year-old man was admitted to our hospital complaining of shortness of breath, weakness, hyperhidrosis and intermittent hypoglycemia of 1-year duration without gross hematuria or lumbago. Imaging studies revealed two masses manifesting as inhomogeneous, with soft-tissue density and having no clear boundaries with the kidney. The patient was initially diagnosed with spaceoccupying lesions of the left kidney (with suspicions of renal cell carcinoma) and left radical nephrectomy was performed. Histologically, the tumors consisted of ovoid or spindle cells, and a hemangiopericytoma-like pattern and cellular atypia was observed in certain areas. Mitotic figures were more than 4 per 10 high-power fields. Immunohistochemically the tumor cells were positive for CD34, CD99 and vimentin. Accordingly, a diagnosis of malignant solitary fibrous tumor of the left kidney (low-grade malignance) was established. Postoperative follow-up of 10 months did not find tumor recurrence or metastasis and hypoglycemia was resolved with the removal of the tumors.
\end{abstract}

\section{Introduction}

Solitary fibrous tumors (SFTs) have been reportedly observed mainly in the pleura but occurrences in urogenital locations including the kidney, prostate and bladder have also been been reported in recent years (1-4). Solitary fibrous tumors arising in the kidney were first described in 1996 by Gelb et al (2); however, few cases, particularly those involving malignancy,

Correspondence to: Professor Ruifa Han, Department of Urology, Second Hospital of Tianjin Medical University, Tianjin Institute of Urology, 23 Pingjiang Road, Hexi, Tianjin 300211, P.R. China E-mail: han_ruifa@hotmail.com

Key words: kidney, malignant, mesenchymal tumor, solitary fibrous tumor have been reported in the worldwide literature to date. We herein describe a case of two malignant SFTs arising in one kidney and the diagnosis which was made mainly based on characteristic histologic findings and immunohistochemical features. We consider that this study will play a significant role in guiding clinical practice on the diagnosis and therapy of renal malignant solitary fibrous tumors.

The study was approved by the ethics committee of Second Hospital of Tianjin Medical University, Tianjin Institute of Urology, Tianjin, China. Informed consent was obtained from the patient prior to the study.

\section{Case report}

A 56-year-old man was admitted to the Second Hospital of Tianjin Medical University complaining of shortness of breath, weakness, hyperhidrosis and intermittent hypoglycemia of 1-year duration without gross hematuria or lumbago. Physical examination revealed no obvious tenderness or percussion pain in the renal regions. Laboratory examinations of routine blood and urine tests revealed no abnormalities. Blood biochemistry including renal and liver function tests were normal except for the blood glucose concentration, which was $2.97 \mathrm{mmol} / \mathrm{l}$. A computed tomography scan revealed the presence of two renal masses presenting as soft tissues in the upper and lower pole of the left kidney, measuring 10x8x7 cm and 10x7x7 cm, respectively. The lesions presented with an inhomogeneous density and had no clear boundary, and exhibited slight peripheral enhancement with contrast medium; however, the center enhanced unequally. Magnetic resonance imaging also revealed two renal masses in the upper and lower pole of the left kidney, which presented as elliptical and inhomogeneous densities (Fig. 1).

An initial diagnosis of space-occupying lesions of the left kidney was given (with suspicions of renal cell carcinoma) and subsequently, the patient underwent left radical nephrectomy. Surgery revealed two masses in the upper and lower pole, with the lower mass showing no clear boundary with the kidney. The cut surface of the tumors was grey to white and crisp in texture with local hemorrhage and necrosis.

Microscopically, both tumors had a similar histological appearance, consisting of ovoid or spindle cells arranged as bundles, swirls or irregularities. Partial focal areas containing vessels were present, which had a hemangiopericytoma-like pattern. Certain areas in the tumors (particularly 


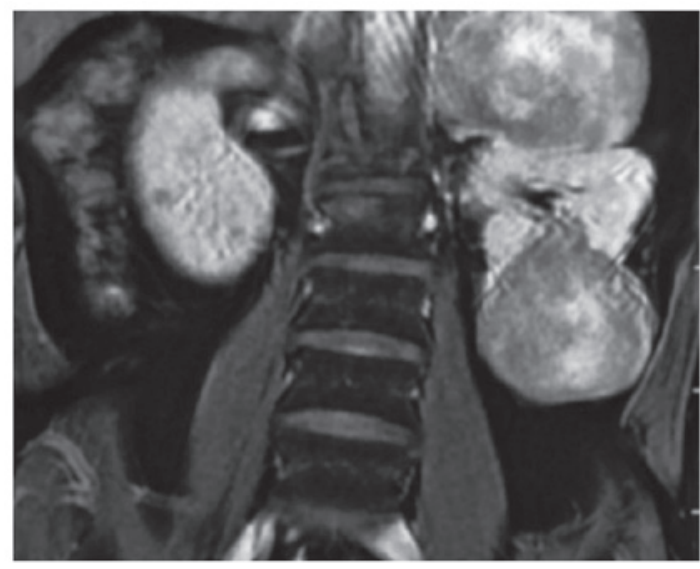

Figure 1. Magnetic resonance imaging revealing two renal masses in the upper and lower pole of the left kidney, which presented as elliptical and inhomogeneous densities.
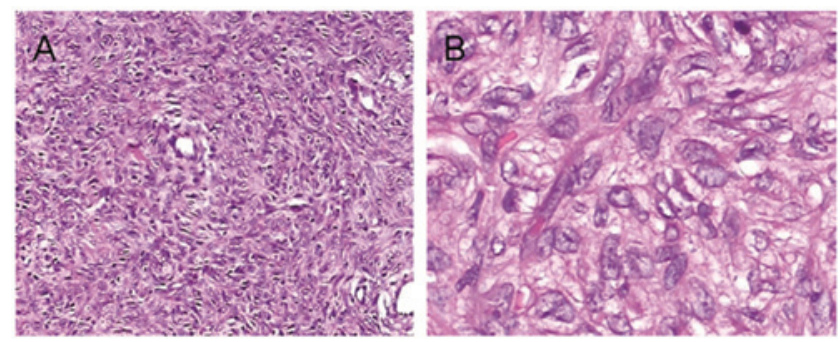

Figure 2. Microscopic features of the malignant solitary fibrous tumor of the kidney. Hematoxylin-eosin stained specimen from the left kidney, showing the tumor consisted of spindle or ovoid cells and a hemangiopericytoma-like pattern. Cellular atypia can be seen in some areas. (A) original magnification x200; (B) original magnification x400.

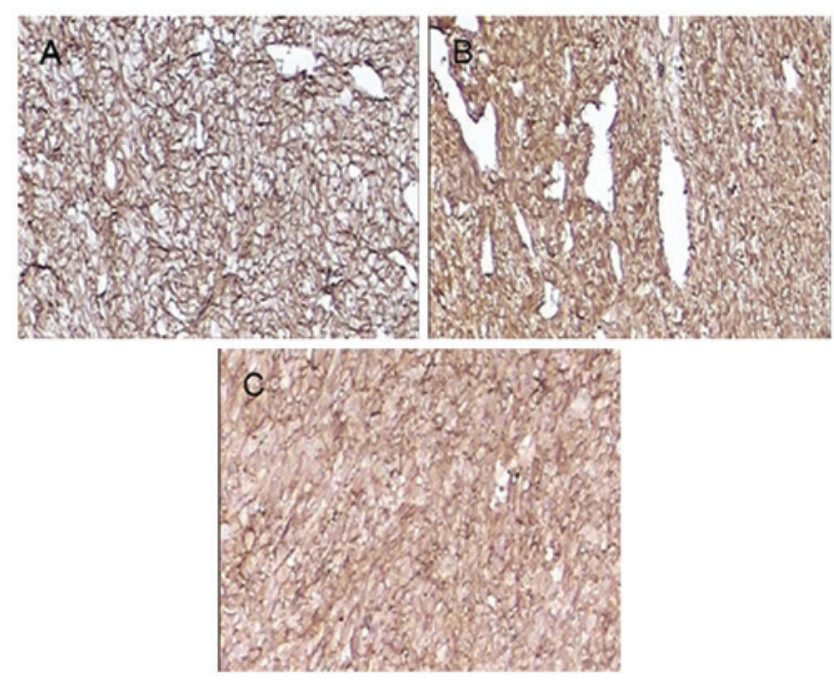

Figure 3. Immunohistochemistry of the malignant solitary fibrous tumor of the kidney. The tumor cells were diffusely positive for (A) CD34, (B) vimentin and (C) CD99.

the lower one) demonstrated increased cells and cellular atypia and mitotic figures were also focally observed (more than 4 per 10 high-power fields). The edge of the mass demonstrated infiltrating growth, and certain areas revealed hemorrhage and/or necrosis (Fig. 2). Immunohistochemically, the tumor cells were positive for CD34, vimentin and CD99 (Fig. 3). Staining for cytokeratin, $\alpha$-smooth muscle actin ( $\alpha$-SMA) and S-100 was negative.

Accordingly, a pathological diagnosis of malignant solitary fibrous tumor of the left kidney (low-grade malignancy) was established based on the microscopic features and the immunohistochemical findings.

A postoperative follow-up of 10 months revealed no signs of tumor recurrence or metastasis and the hypoglycemia was resolved with the removal of the tumors.

\section{Discussion}

Solitary fibrous tumors arising in the kidney were first described in 1996 by Gelb et al (2). The majority of renal SFTs are observed in adults in a wide age range (18-85); however, one pediatric case has been reported in a 4-year-old boy (5). In general, SFTs are slow-growing tumors with a favorable prognosis, although there have been some malignant cases (6).

Symptoms of SFTs usually do not differ from those reported by patients with renal cell carcinoma, which include flank or abdominal pain and/or gross hematuria. In certain patients, the tumor is found incidentally and in others the mass is palpable $(2,6)$. Non islet-cell hypoglycemia (Doege Potter syndrome) arises due to the production of high molecular weight insulin-like growth factors and is a frequently associated paraneoplastic syndrome, which may have malignant behavior or poor survival from diagnosis (7). In our case, the patient had symptoms of hypoglycemia; however, it was resolved with the removal of the neoplasm suggesting it was the result of a paraneoplastic syndrome.

Macroscopically, renal SFTs are usually single; however, this was not observed in the present case. Occasionally, myxoid and pseudocapsules are also present. The size of the tumors is usually between 2.0 and $25.0 \mathrm{~cm}$. The majority of cases are without cystic, hemorrhagic or necrotic changes which may be malignant (6). Microscopically, SFTs of the kidney are pathologically characterized by spindle cell proliferation showing a patternless architecture with a combination of alternating hypocellular and hypercellular areas. Furthermore, SFTs are tumors with variable cellularity and are composed of a mixture of haphazard, storiform, short fascicular arrangements of bland spindle cells and collagenous bands. They are highly vascular tumors, with hemangiopericytoma-like patterns observed in certain areas. Myxoid changes and fibrosis may also be observed $(6,8,9)$.

Immunohistochemical study is key to diagnosis. Although it is not specific to SFTs, CD34 immunoreactivity has been reported to be diffusely expressed in many cases of SFTs, thus strong CD34 reactivity is currently regarded as the most prominent characteristic and indispensable finding to aid diagnosis (2-4,8-10). In addition, a positive expression of bcl-2, vimentin and CD99 is also present in the majority of SFTs; however, cytokeratin, $\alpha$-smooth muscle actin ( $\alpha$-SMA) and S-100 are usually absent (2-4,8-10).

The majority of SFTs are benign; however, a few have revealed histologically malignant features. The criteria for malignant SFTs, first proposed by England et al (10) in 1989, include increased cellularity with crowded/overlapping nuclei, 
cellular pleomorphism and a mitotic count of more than 4 per 10 high-power fields. However, in spite of cytologic malignancy in SFTs, the clinical outcome is not always poor (10). In the present case, the majority of areas in the tumors (particularly the lower one) demonstrated increase cell density and cellular atypia, and mitotic figures were more than 4 per 10 high-power fields. The edge of the masses showed infiltrating growth and certain areas revealed hemorrhage or necrosis. Accordingly, the diagnosis was confirmed as malignant.

Solitary fibrous tumors should be distinguished from benign and malignant spindle cell tumors of the kidney. Inflammatory myofibroblastic tumors are characterized by spindle cells in an edematous background with blood vessels and inflammation consisting of lymphocytes and plasma cells. Furthermore, a mixture of spindle cells and collagenization with CD34 reactivity differentiates SFTs from inflammatory myofibroblastic tumors. Fibromas are small tumors that do not react with CD34. Leiomyoma can be excluded by the lack of expression of muscle markers. The absence of smooth muscle and adipose tissue components, with the aid of HMB-45 immunonegativity, distinguishes SFT from angiomyolipoma. It is occasionally difficult to differentiate hemangiopericytoma from SFT; however, the presence of spindle cell cytological features, various growth patterns with alternating hypocellular and hypercellular areas and abundant collagenization favors a diagnosis of SFTs. Solitary fibrous tumors may also be differentiated from leiomyosarcoma, malignant nerve sheath tumors, sarcomatoid renal and transitional cell carcinoma by the absence of dense cellularity, severe atypia, high mitotic rates and with the help of immunohistochemical staining of these tumors for $\alpha$-smooth muscle actin, S-100 and cytokeratin (8).

Surgery is considered the first choice of treatment if possible. The use of chemotherapy is reserved for metastatic or symptomatic non-resectable SFTs; however, there are still no standard chemotherapeutic indications or regimens. The relatively effective drugs appear to be those which are commonly used in soft tissue sarcomas even though SFTs are considered chemoresistant $(11,12)$. Radiation therapy is of some benefit, when applicable, given in combination with chemotherapy (13). The novel targeted drug imatinib mesilate, which exerts some activity on SFTs expressing the wild-type PDGFR- $\beta$, has recently been reported $(14,15)$.

The prognosis is generally good for SFTs and the majority are non-recurring and non-metastazing tumors. It is estimated that 10 to $15 \%$ of intrathoracic SFTs and $10 \%$ of extrathoracic SFTs will recur and/or metastasize $(16,17)$, therefore SFT is regarded as an 'intermediate malignant, rarely metastasizing neoplasm' (18). Poor prognosis of SFTs may occur when there is incomplete resection, a large size $(>10 \mathrm{~cm})$, presence of malignant cellularity or they are located outside the thoracic cavity. An additional factor conferring a worse prognosis in SFTs is dedifferentiation or sarcomatous overgrowth, which represents an abrupt transition to a morphologically anaplastic component (19). In the present case, a postoperative follow-up of 10 months revealed no recurrence or metastasis. Nevertheless, long-term follow-up is indispensable since metastases may still occur after several years.

To summarize, we presented a case of two malignant SFTs in one kidney with characteristic clinicopathological features.
Although malignant SFTs in extrapleural sites remain extremely uncommon, it is indispensable to consider this possibility when renal spindle cell tumors are encountered. In view of characteristic findings which are often not observed in imaging studies including CT and MRI, the histologic features and immunohistochemical staining for CD34, CD99, bcl-2 and vimentin may be helpful in confirming the diagnosis of malignant SFTs of the kidney.

\section{References}

1. Robinson L: Solitary fibrous tumor of pleura. Cancer Control 13: 264-269, 2006.

2. Gelb A, Simmons M and Weidner N: Solitary fibrous tumor involving the renal capsule. Am J Surg Pathol 20: 1288-1295, 1996.

3. Marzi M, Pira P, D'Alpaos M, Paiusco A, Canessa S, Minervini MS and Di Zitti P: The solitary fibrous malignant tumor of the kidney: clinical and pathological considerations on a case revisiting the literature. Minerva Urol Nefrol 63: 109-113, 2011.

4. Talvitie H, Aström K, Larsson O, et al: Solitary fibrous tumor of the prostate: A report of two cases. Pathol Int 61: 536-538, 2011.

5. Ferrari $\mathrm{N}$ and Nield L: Final diagnosis: solitary fibrous tumor of the kidney. Clin Pediatr (Phila) 45: 871-873, 2006.

6. Fine SW, McCarthy DM, Chan TY, et al: Malignant solitary fibrous tumor of the kidney: report of a case and comprehensive review of the literature. Arch Pathol Lab Med 130: 857-861, 2006.

7. Herrmann B, Saller B, Kiess W, Morgenroth K, Drochner K and Schröder T: Primary malignant fibrous histiocytoma of the lung: IGF-II producing tumor induces fasting hypoglycaemia. Exp Clin Endocr Diab 108: 515-518, 2000.

8. Magro G, Cavallaro V, Torrisi A, Lopes M, Dell'Albani M and Lanzafame S: Intrarenal solitary fibrous tumor of the kidney report of a case with emphasis on the differential diagnosis in the wide spectrum of monomorphous spindle cell tumors of the kidney. Pathol Res Pract 198: 37-43, 2002.

9. Yazaki T, Satoh S, Iizumi T, Umeda T and Yamaguchi Y: Solitary fibrous tumor of renal pelvis. Int J Urol 8: 504-508, 2001.

10. England D, Hochholzer L and McCarthy M: Localized benign and malignant fibrous tumors of the pleura: a clinicopathologic review of 223 cases. Am J Surg Pathol 13: 640-658, 1989.

11. Grobmyer SR, Maki RG, Demetri GD, Mazumdar M, Riedel E, Brennan MF, et al: Neo-adjuvant chemotherapy for primary high-grade extremity soft tissue sarcoma. Ann Oncol 15: 1667-1672, 2004.

12. Demetri GD, Antonia S, Benjamin RS, et al: NCCN Clinical Practice Guidelines in Oncology: Soft Tissue Sarcoma-V. 2. 2009.

13. De Boer J, Jager PL, Wiggers T, Nieboer P, Machteld WAN, Pras E, et al: The therapeutic challenge of a nonresectable solitary fibrous tumor in a hypoglycemic patient. Int J Clin Oncol 11: 478-481, 2006

14. Prunotto M, Bosco M, Daniele L, Macri' L, Bonello L, Schirosi L, et al: Imatinib inhibits in vitro proliferation of cells derived from a pleural solitary fibrous tumor expressing platelet-derived growth factor receptor-beta. Lung Cancer 64: 244-246, 2009.

15. De Pas T, Toffalorio F, Colombo P, Trifirò G, Pelosi G, Vigna PD, et al: Brief report: activity of imatinib in a patient with platelet-derived-growth-factor receptor positive malignant solitary fibrous tumor of the pleura. J Thorac Oncol 3: 938-941, 2008.

16. Weiss SW and Goldblum JR (eds): Soft tissue tumors of intermediate malignancy of uncertain type. In: Soft Tissue Tumor. 5th edition. Mosby Elsevier, Philadephia, pp1093-1160, 2008.

17. Vallat-Decouvelaere AV, Dry SM and Fletcher CD: Atypical and malignant solitary fibrous tumors in extrathoracic locations: evidence of their comparability to intra-thoracic tumors. Am J Surg Pathol 22: 1501-1511, 1998.

18. Guillou LFJ, Fletcher CDM and Mandahi N: Extrapleural solitary fibrous tumour and hemangiopericytoma. In: World Health Organization Classification of Tumours: Pathology and Genetics of Tumours of Soft Tissue and Bone. Fletcher. CDM, Unni KK and Mertens F (eds). IARC Press, Lyon, pp86-90, 2002.

19. Mosquera JM and Fletcher CD: Expanding the spectrum of malignant progression in solitary fibrous tumors: a study of 8 cases with a discrete anaplastic component - is this dedifferentiated SFT? Am J Surg Pathol 33: 1314-1321, 2009. 